\title{
Production of Lovastatin by Penicillium spp. Soil Microfungi
}

\author{
Emine Seydametova, Member, APCBEES, Jailani Salihon, Norazwina Zainol, and Peter Convey
}

\begin{abstract}
Lovastatin is an important fungal secondary metabolite inhibiting the enzyme which catalyzes a rate-limiting step in the biosynthesis of cholesterol. As microfungi of the genus Penicillium are active producers of cholesterol lowering agents (statins), studies of this genus have gained considerable momentum. In Malaysia, both agricultural soils and those of natural ecosystems are valuable sources of filamentous fungi, whose biotechnological potential in terms of lovastatin production has not been well investigated. The aim of the present study is to determine lovastatin-producing potential of Penicillium spp. microfungi isolated from soil in Pahang State (Malaysia). Fungal cultures were isolated, purified, and classified based on characters of colony morphology and microscopic features. All identified representatives of Penicillium were tested for their ability to produce lovastatin. Isolates were cultivated in submerged fermentation. High-performance liquid chromatography (HPLC) was used for detection of lovastatin. Among 54 fungal cultures examined, four Penicillium strains were confirmed to produce this statin. The isolate Penicillium sp. ESF2M was the strongest lovastatin producer, with a yield of $20 \mathrm{mg} / \mathrm{l}$. This strain should now be subjected to a further strain improvement program to maximize the yield of this important statin.
\end{abstract}

Index Terms—Fermentation, lovastatin, Penicillium spp.

\section{INTRODUCTION}

The last century has witnessed the discovery, isolation and chemical characterization of a wide diversity of natural bioactive products from different species of filamentous fungi. However, members of the genus Penicillium have been of particular interest to the research community because of their biotechnological potential.

Among the secondary metabolites known to be produced by Penicillium spp. are statins. These are a group of extremely successful drugs that lower the cholesterol level in blood, decreasing the risk of heart attack and other cardiovascular problems. The cholesterol biosynthetic pathway, starting from acetyl-CoA, involves more than 25 enzymes, but the rate-limiting step is the conversion of 3-hydroxy-3-methylglutaryl-coenzyme A (HMG-CoA) to mevalonate by HMG-CoA reductase. Statins can inhibit

Manuscript received August 20, 2012; revised September 10, 2012.

This work was financially supported by the Universiti Malaysia Pahang (UMP) under short-term Grants: GRS090334 and RDU100319.

Emine Seydametova, Jailani Salihon, and Norazwina Zainol are with Faculty of Chemical and Natural Resources Engineering, Universiti Malaysia Pahang (UMP), Kuantan, 26300 Malaysia, on leave from the Institute of Microbiology under Academy of Sciences of Uzbekistan, Tashkent, Uzbekistan (e-mail: e_seydametova@ yahoo.com, jailani@ump.edu.my, azwina@ump.edu.my).

Peter Convey is with British Antarctic Survey, Cambridge CB3 0ET,
HMG-CoA reductase activity [1]. The mechanism involved in the control of endogenous cholesterol levels makes these molecules suitable for therapeutic use [2]-[6].

Lovastatin (also known as monacolin $\mathrm{K}$, mevinolin) is a natural statin that shows significant hypocholesterolemic effects. Several fungal genera including Aspergillus, Penicillium and Trichoderma have been reported to be able to produce lovastatin [7], [8]. The results of screening experiments reported by Shindia [7] assessed Aspergillus terreus as the best lovastatin-producing fungus, exhibiting a capacity to synthesize it at a production level of $84 \mathrm{mg} / \mathrm{l}$. Penicillium citrinum and Trichoderma viride were also able to produce the compound in concentrations of 61 and 4.1 $\mathrm{mg} / \mathrm{l}$, respectively. The following fungi have also been recognized as lovastatin producers - A. terreus $(55 \mathrm{mg} / \mathrm{l})$, Penicillium funiculosum (pinophilum) $(19.3 \mathrm{mg} / \mathrm{l})$ and $T$. viride $(9 \mathrm{mg} / \mathrm{l})[8]$.

Currently, lovastatin is produced commercially by active mutant strains of $A$. terreus. However, at present the industrial biotechnology field has utilized only a very small proportion of the natural microbial arsenal in the search for new producers of statins. Therefore, the cholesterol-lowering activity of some secondary metabolites from Penicillium species suggests that this genus is worthy of further investigation. Soil has been recognized as a reservoir for a wide variety of filamentous fungi, and many soil fungi are representatives of the rapidly growing and nutritionally non-specialized genus Penicillium. The present investigation was therefore undertaken to examine the potential of soil Penicillium spp. to produce lovastatin.

\section{Materials AND MethodS}

\section{A. Chemicals}

Potato-dextrose agar (PDA) and malt extract agar (MEA) were obtained from Merck, Germany. Chemical reagents required for the culture media, including glucose, sucrose, glycerol, peptone, yeast extract, $\mathrm{MgSO}_{4} \times 7 \mathrm{H}_{2} \mathrm{O}, \mathrm{NaNO}_{3}, \mathrm{KCl}$, $\mathrm{K}_{2} \mathrm{HPO}_{4} \times 3 \mathrm{H}_{2} \mathrm{O}, \mathrm{FeSO}_{4} \times 7 \mathrm{H}_{2} \mathrm{O}, \mathrm{CuSO}_{4} \times 5 \mathrm{H}_{2} \mathrm{O}, \mathrm{ZnSO}_{4} \times 7 \mathrm{H}_{2} \mathrm{O}$, $\mathrm{NaOH}, \mathrm{HCl}$, agar-agar were obtained from R\&M Chemicals, UK. Reagents used for extraction, ethyl alcohol (R\&M Chemicals, UK) and ethyl acetate (Merck, Germany), were of analytical grade. The pure lovastatin (99.7\%) used as a standard in this study was obtained from Merck, Germany.

\section{B. Soil Sampling}

Several sampling stations in Pahang State (Malaysia) were selected (Table I). Twenty-one sites were sampled in total, in Gambang (10 sites), Kuantan (3 sites), Balok (4 sites), and Cherating (4 sites). At each site, samples of soil were taken at 
depths of 10-50 cm, packed into labeled sterilized polyethylene bags using a sterilized spatula, and returned to the laboratory for further examination. A total of 63 soil samples were collected in September and October 2009.

\begin{tabular}{lll}
\multicolumn{2}{c}{ TABLE I: SOIL SAMPLES OBTAINED FROM SAMPLING STATIONS } \\
\hline \hline \multirow{2}{*}{ Sampling station } & Depth, cm & $\begin{array}{l}\text { Number of soil } \\
\text { samples }\end{array}$ \\
\hline Gambang & 10 & 9 \\
& 20 & 9 \\
& 30 & 6 \\
& 40 & 3 \\
Kuantan & 50 & 3 \\
Balok & 10 & 9 \\
Cherating & 10 & 12 \\
\hline \hline
\end{tabular}

\section{Isolation and Identification of Penicillium spp}

Filamentous fungi were initially isolated by plating collected soil samples on PDA. Individual colonies of microfungi were picked and purified by streaking onto fresh PDA medium. The pure fungal isolates were kept on PDA medium at $4^{\circ} \mathrm{C}$ and re-cultured every 4 weeks. The fungal isolates were transferred to appropriate agar media for identification: Czapek-Dox agar (CZ), Czapek yeast extract agar (CYA), yeast extract sucrose agar (YES), and MEA. Isolates were identified morphologically following Pitt [9]. The macroscopic features examined included colony diameter, obverse and reverse colony colors, and presence of exudate droplets. Microscopic characteristics such as conidiophore branching patterns were examined using an optical microscope (Primo Star Carl Zeiss, Germany). These techniques allowed the fungal isolates to be identified to the genus level.

\section{Fermentation Procedure}

Fifty-four Penicillium spp. isolates were obtained and tested for their ability to produce lovastatin. The fungal strains were maintained on PDA slants and incubated at $25^{\circ} \mathrm{C}$ for 7 days. A spore suspension was then prepared by suspending the spores from the slant in $10 \mathrm{ml}$ of sterilized distilled water. Ten percent of the spore suspension prepared from each slant was used to inoculate $20 \mathrm{ml}$ of sterile screening medium. Shake flask fermentation was carried out for each fungal culture in 100 -ml flasks at $25^{\circ} \mathrm{C}, 180 \mathrm{rpm}$, for 7 days in an incubator shaker (Multitron $\mathrm{II}{ }^{\circledR}$ Version 2; Inforce, Switzerland).

\section{E. Lovastatin Determination}

Ethyl acetate extracts of fermentation broths obtained according to the extraction procedure described by Samiee et al. [8] were used for high-performance liquid chromatography (HPLC) analysis of lovastatin presence. Five $\mu \mathrm{l}$ from the organic phase were injected on a $250 \times 4.6 \mathrm{~mm}$ ID Zorbax Eclipse Plus C18 column, 5 $\mu \mathrm{m}$ particle size. The mobile phase consisted of acetonitrile and water $(60: 40, \mathrm{v} / \mathrm{v})$ with a $\mathrm{pH}$ value adjusted to $\mathrm{pH} 3 \pm 0.2$ by addition of $\mathrm{H}_{3} \mathrm{PO}_{4}$. The flow rate was maintained at 0.8 $\mathrm{ml} / \mathrm{min}$ and the presence of lovastatin was confirmed by measurement at $238 \mathrm{~nm}$ [7]. Pure lovastatin (99.7\%, Merck, Germany) was used as a standard.

\section{F. Dry Cell Weight Estimation}

Dry cell weight (DCW) was estimated by filtering the content of each flask through a pre-weighed Whatman filter paper No. 41. The collected mycelial residues were washed with distilled water and dried at $105^{\circ} \mathrm{C}$ until constant weight.

\section{G. Statistical Analysis}

Each experiment was conducted in three replicates. Mean values with standard deviation are presented.

\section{RESUlTS AND DISCUSSION}

\section{A. Morphological Characterization of Penicillium spp}

A total of 54 fungal cultures were isolated from the collected soil samples. The morphological properties showed variability between the different cultures and nutrient media used. Colony diameters of the isolates varied on different media: 4-20 mm (CZ), 19-44 mm (CYA), 25-50 mm (YES), and 14-60 mm (MEA). In addition, the cultures obtained had different obverse and reverse colony colors. In general, almost all fungal isolates had white to cream obverse and pale reverse colony color on CZ medium. Colonies on CYA medium were white to cream, yellow, glaucous, or dark green on the obverse, whereas on the reverse they were - pale, yellow, orange, fawn, and dark brown. On YES medium both obverse and reverse colony colors of isolates varied from white and cream to dark green and from pale to fawn, respectively. Most isolates on MEA medium had obverse colony colors of white to cream, yellow, or dark green, with the colony reverse being yellow, orange or fawn. These are consistent with the observations of Pitt [9].

Almost all the fungal cultures obtained did not produce exudates on CZ and YES media. The presence of uncolored, yellow, brown, and dark brown exudates was observed on CYA and MEA media. Some Penicillium species are known to produce distinct exudate droplets, for instance, yellow in Penicillium chrysogenum and dark brown in Penicillium venetum [10].

Among the fungal isolates obtained there were examples with both simple and branched conidiophores with metulae, phialides and conidia, features which are observed amongst Penicillium spp. [9]. The appearance of the spore head resembles that of a brush, and the spore head is termed a penicillus, which is Latin for a brush. Thus, microscopic features of the isolates obtained indicated that they were characterized by conidiophores and microscopic elements typical of the genus Penicillium.

TABLE II: LOVASTATIN-PRODUCING PENICILLIUM STRAINS

\begin{tabular}{lll}
\hline \hline Fungal culture & $\begin{array}{l}\text { Lovastatin, } \\
\mathrm{mg} / \mathrm{l}\end{array}$ & Lovastatin, $\mu \mathrm{g} / \mathrm{g} \mathrm{DCW}$ \\
\hline Penicillium sp. ESF2M & $20.39 \pm 0.87$ & $1952.72 \pm 170.56$ \\
Penicillium sp. ESF5P & $7.10 \pm 0.35$ & $536.65 \pm 54.49$ \\
Penicillium sp. ESF11P & $2.12 \pm 0.13$ & $288.20 \pm 6.29$ \\
Penicillium sp. ESF19P & $3.86 \pm 0.17$ & $421.22 \pm 14.45$
\end{tabular}

Results are presented as mean \pm SD from three replicates

\section{B. Screening of Lovastatin-Producing Penicillium spp}

Fifty-four strains were screened for lovastatin production 
using HPLC analysis after extraction of this compound from the fermentation broths with ethyl acetate. Of the screened strains only four isolates, Penicillium sp. ESF2M, Penicillium sp. ESF5P, Penicillium sp. ESF11P and Penicillium sp. ESF 19P, were confirmed as producers of lovastatin (Table II), with no production detected in any other isolates.

The lovastatin standard showed a retention time of 19.1 min under the HPLC column elution conditions used (Fig. 1(a)). Lovastatin quantification was carried out on Penicillium spp. extracts from culture broths. Among positive isolates, Penicillium sp. ESF2M was identified as the best lovastatin-producing strain (Fig. 1(b)), with a production level of $20.39 \mathrm{mg} / \mathrm{l}$. The remaining lovastatin producers achieved considerably lower concentrations.

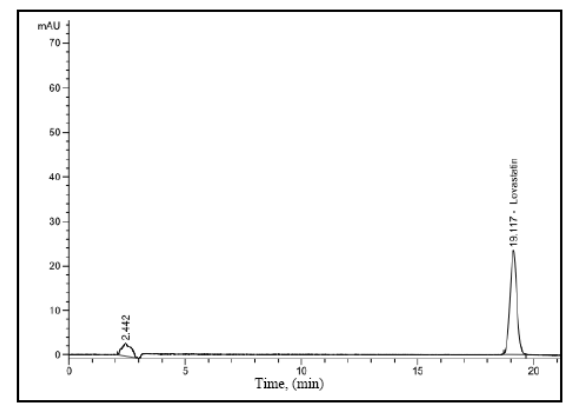

a)

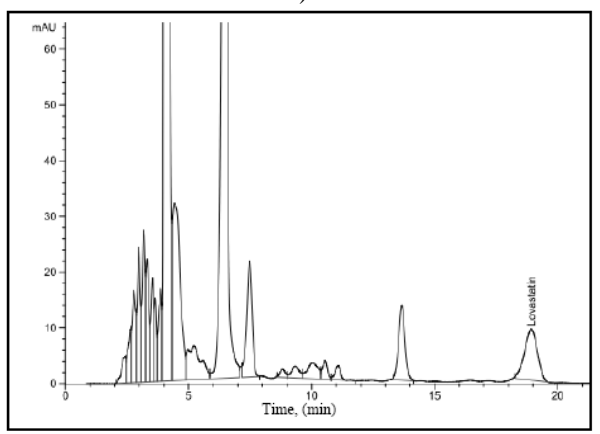

b)

Fig. 1. High-performance liquid chromatograms: (a) lovastatin standard, (b) ethyl acetate extract of Penicillium sp. ESF2M.

The yield of lovastatin from the isolate Penicillium sp. ESF $2 \mathrm{M}$ was comparable with reported production levels in other species of Penicillium [7], [8]. Shindia [7] reported a somewhat higher production level by wild $P$. citrinum strain (61 mg/l) isolated from soil, while Samiee et al. [8] showed that $P$. funiculosum (pinophilum) produced this statin at a very similar level $(19.3 \mathrm{mg} / \mathrm{l})$ to the strain selected in present study.

The isolate Penicillium sp. ESF2M also demonstrated the highest yield of lovastatin per gram dry cell weight (1952.72 $\mu \mathrm{g} / \mathrm{g})$. These data support the use of Penicillium sp. ESF2M in further research in order to enhance its productive capability.

\section{CONCLUSION}

Soils in Pahang State are a valuable source of microfungi from the genus Penicillium. A total of 54 Penicillium spp. strains were isolated from soil samples in this study and examined for lovastatin production. Four of these strains were confirmed to produce lovastatin, with the isolate
Penicillium sp. ESF2M being recommended for further studies as a potent lovastatin producer, comparable with strains reported in the literature.

\section{REFERENCES}

[1] M. Manzoni and M. Rollini, "Biosynthesis and biotechnological production of statins by filamentous fungi and application of these cholesterol-lowering drugs," Appl Microbiol Biotechnol, vol. 58, pp. 555-564, 2002.

[2] M. Buemi, M. Senatore, F. Corica, C. Aloisi, A. Romeo, E. Cavallaro, F. Floccari, D. Tramontana, and N. Frisina, "Statins and progressive renal disease,” Med Res Rev, vol. 22, pp. 76-84, 2002.

[3] P. H. Chong, J. D. Seeger, and C. Franklin, "Clinically relevant differences between the statins: implications for therapeutic selection,” Am J Med, vol. 111, pp. 390-400, 2001.

[4] G. P. Eckert, W. G. Wood, and W. E. Muller, "Statins: drugs for Alzheimer's disease?,” J Neural Transm, vol. 112, pp. 1057-1071, 2005.

[5] C. D. Furberg, "Natural statins and stroke risk," Circulation, vol. 99, pp. 185-188, 1999.

[6] D. J. Maron, S. Fazio, and M. F. Linton, "Current perspective on statins," Circulation, vol. 101, pp. 207-213, 2000.

[7] A. A. Shindia, "Mevinolin production by some fungi," Folia Microbiol, vol. 42, no. 5, pp. 477-480, 1997.

[8] S. M. Samiee, N. Moazami, S. Haghighi, F. A. Mohseni, S. Mirdamadi, and M. R. Bakhtiari, "Screening of lovastatin production by filamentous fungi,” Iran Biomed J, vol. 7, no. 1, pp. 29-33, 2003.

[9] J. I. Pitt, the Genus Penicillium and Its Teleomorphic States Eupenicillium and Talaromyces, London, U.K. Academic Press, 1979.

[10] K. B. Raper and C. Thom, a Manual of the Penicillia, Baltimore, Maryland: Williams and Wilkins Co., 1949.

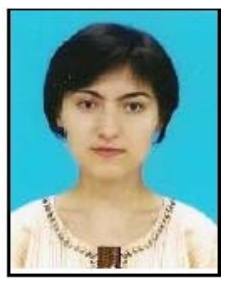

Emine Seydametova is a Researcher of the Institute of Microbiology, Academy of Sciences of Uzbekistan. Currently, she does her research in bioprocess development as a Ph.D. candidate of the Faculty of Chemical and Natural Resources Engineering, Universiti Malaysia Pahang. She received her B.Sc. in microbiology and M.Sc. in biochemistry from National University of Uzbekistan. Her research interests focus on the production of microbial metabolites. She has published five papers and one monograph.

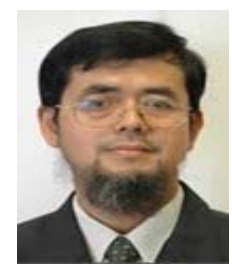

Jailani Salihon is the Dean of the Faculty of Chemical and Natural Resources Engineering, Universiti Malaysia Pahang. He completed his Bachelor degree in chemical engineering in 1980 at Loughborough University of Technology, UK. He received his Doctorate in chemical engineering in 1984 from University of Surrey, UK. His research area is fermentation process development. He has published 16 papers.

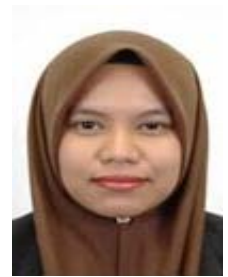

Norazwina Zainol is a Senior Lecturer of the Faculty of Chemical and Natural Resources Engineering, Universiti Malaysia Pahang. She received her Bachelor, Master and Ph.D. degrees in chemical engineering and process from Universiti Kebangsaan Malaysia. Her research area is environmental biotechnology.

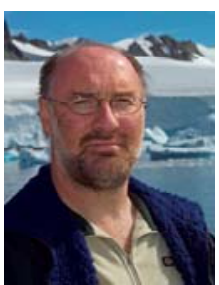

Peter Convey is a Senior Biologist of the British Antarctic Survey, UK. He has wide interests in biodiversity and ecosystems structure and functions, including the microbial components of soil ecosystems and their interaction 